\title{
STABILITY OF CYCLES
}

\section{AND EXPECTATIONS}

Jean-Michel GRANDMONT and Guy LAROQUE

$N^{\circ} 8519$

April 1985

Revised Jury 1985 
Grandmont, Jean-Michel ; Laroque, Guy - Stability of Cycles and Expectations

The actual dynamics of an economy depends on how agents forecast the future at every date as a function of their information on the past, while possibly learning the structure of their environment. We show in the case of a onedimensional state variable that under mild conditions on expectations functions, a given cycle with perfect foresight that is stable in the actual dynamics is stable in a fictitious backward perfect foresight dynamics. We exhibit a restricted class of expectations functions for which the converse is true. J. Econ. Theory, 1986, ,pp. (English). Grandmont: CEPREMAP, Paris ; Laroque : INSEE, Paris.

Journal of Economic Literature Classification Numbers : 021,022 , 023,131

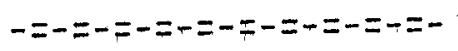

RES U ME

La dynamique en temps réel d'une économie dépend de la façon dont les agents prévoient à tout moment le futur en fonction de leur information sur le passé. Ce cadre permet de plus d'incorporer un apprentissage possible des agents. Nous montrons dans le cas d'une variable d'état unidimensionnelle que sous des conditions minimes sur les fonctions d'anticipations, un cycle donré avec anticipations correctes qui est stable dans la dynamique en temps réel est stable dans une dynamique fictive avec prévision parfaite dans laquelle le temps va décroissant. Nous mettons en évidence une classe de fonctions d'anticipations pour laquelie la réciproque est vraie. 


\section{STABILITY OF CYCLES AND EXPECTATIONS}

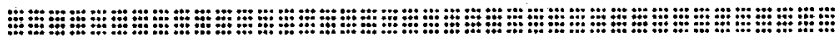

Jean-Miche 1 GRANDMONT ${ }^{*}$ and GuY LAROQUE ${ }^{\star \star}$

An important feature of economic systems is that the state of the economy at a given date depends in an essential way on the agents' expectations about the future. The actual dynamics followed by the economy is thus determined by the way in which the agents forecast the future as a function of their information on the past at every date, while possibly learning the structure of their environment.

In the long run, the economy may converge to a steady state or a cycle along which the agents do not make forecasting errors. It is we 11 known however that there may exist a large number of steady states or cycles with perfect foresight. The question studied in the present paper is whether one can single out those cycles with perfect foresight that are asymptotically stable in the actual dynamics, although little is known about the expectations formation process. Attention is focussed on the simple case in which the state of the economy can be described at each date by a real number, as for instance in simple versions of the over lapping generations model.

Cycles with perfect foresight may be alternatively viewed as periodic trajectories of a fictitious perfect foresight dynamics in which time goes backward (a backward perfect foresight (b.p.f.) dynamics), the state of the economy at $t+1$, being by assumption correctly anticipated, determining the state at $t$. We show that under rather general conditions on expectations functions, a given cycle with perfect foresight that is stable in the actual dynamics is necessarily stable in the b.p.f. dynamics. Simple examples show that the converse is not generally true. Yet we exhibit, in a slight generalization of Grandmont [8], a restricted class of expectations functions ensuring that all cycles of a given period that are stable in the b.p.f. dynamics are also stable in the actual dynamics of the economy. 
This set of results shows that one should be very cautious when interpreting the stability results that one gets from dynamical rational expectations models in which time goes forward. Taking into account the agents' learning behaviour on the transition path, as one should, may reverse the stability diagnosis. That sort of qualitative conclusion is comforted by similar (in spirit) studies of rational expectations macroeconomic models under uncertainty ( $P$. Champsaur [3]).

It remains to be seen if the results of this paper carry over qualitatively to more general structures. In particular, it would be important to incorporate "error learning" behaviour as in G. Fuchs [4, 5, 6] and/or to extend this type of analysis to a multidimensional framework.

\section{MODEL AND RESULTS}

An important feature of economic systems is that the agents' forecasts of the future influence in an essential way the current state of the economy. If we consider the simplest case in which the state of the economy can be described by a single real number $x$, a possible formulation of the phenomenon is that the law of motion of the economic system is governed (in discrete time) by

$$
x_{t}=F\left(x_{t+1}^{e}\right)
$$

in which $x_{t}$ is the current state of the system, $x_{t+1}^{e}$ is the state that the agents anticipate at date $t$ (with subjective certainty) for the next date, and $F$ is a map - assumed from now on continuously differentiable - from some open interval $X$ of the real line into itself. That sort of formulation would arise for instance in a simple version of the over lapping generations model.

To describe the evolution of the economy as a dynamical system, i.e. to determine the state of the economy at $t$ as a function of the past, we have to 
specify how the agents form their forecast $x_{t+1}^{e}$ at date $t$ as a function of their information at that date. If one assumes that agents do know at date $t$ the current and past values of the state variable, the forecast $x_{t+1}^{e}$ will be a function at that date of these variables. In order to make the analysis tractable, we shall focus attention on the simple case in which the forecast is a fixed function over time of only the $T$ immediate past values of $x$. This yields

$$
x_{t+1}^{e}=\psi\left(x_{t-1}, \ldots, x_{t-T}\right)
$$

in which the expectation function $\psi$ - assumed from now on continuously differentiable - maps the Cartesian product $x^{\top}$ into $x^{(2)}$.

Equations (1.1) and (1.2) imply that the dynamic evolution of the economy is ruled by the difference equation

$$
x_{t}=W\left(x_{t-1}, \ldots, x_{t-T}\right)
$$

in which the temporary equilibrium function $W=F \circ \Psi$ maps $x^{\top}$ into $X$.

It is natural to try to separate in the dynamics (1.3), the relative role of the structural behavioural relationship $F$ from that of the expectations function. In order to do that, we define an intertemporal equilibrium with perfect foresight as a double ended infinite sequence $\left(x_{t}\right)$ such that

$$
x_{t}=F\left(x_{t+1}\right)
$$

for all $t=0, \pm 1, \ldots$. A periodic equilibrium or a cycle with perfect foresight, with period $k$, is then defined as an intertemporal equilibrium $\left(x_{t}\right)$ such that $x_{t+k}=x_{t}$ for all $t, k$ being the smallest positive integer having this property. 
The expectation function $\psi$ is said to detect period $k$ if for every sequence $\left(x_{t-1}, \ldots, x_{t-T}\right)$ in $x^{\top}$ that has period $k, i . e$. that satisfies $x_{t-j-k}=x_{t-j}$ for all relevant $j$, one has

$$
\begin{array}{rlrl}
\psi\left(x_{t-1}, \ldots, x_{t-T}\right) & =x_{t+1-k} & & \text { if } k \geqslant 2 \\
& =x_{t-1} & \text { if } k=1
\end{array}
$$

Clearly, $\psi$ detects period $k$ if and only if it detects all perfods that divide $k$. Of course, the memory lag $T$ is assumed to be larger than all periods that $\psi$ detects.

It is Immediate to see if $\psi$ detects period $k$, any solution $\left(x_{t}\right)$ of (1.3) that has period $k$ is a cycle with perfect foresight, i.e. it is a solution of (1.4) with the same period - and conversely.

Consider a given cycle $\left(\bar{x}_{t}\right)$ with perfect foresight, with a period $k$ that is detected by the expectation function $\psi$. Our objective is to study the stability of this cycle under the dynamics (1.3), and more specifically, to relate it to the stability of the same cycle under the simpler but entirely fictitious backward perfect foresight (b.0.f.) dynamics (1.4) induced by $F$. Loosely speaking, this cycle is asymptotically stable in the actual dynamics (1.3) - for short $W$-stable - if when one starts at date 0 with initial values $\left(x_{-1}, \ldots, x_{-T}\right)$ close enough to the cycle, the trajectory defined by (1.3) stays close to the cycle and converge actually to it. A similar definition applies to the b.p.f. dynamics of (1.4), in which case we say that the cycle is F-stable (precise mathematical definitions will be given in the next section).

We are now in a position to state our main result. 
5

THEOREM 1.1. Assume that the expectation function $\psi$ detects period $2 k$, and let $\left(\bar{x}_{t}\right)$ be a cycle with perfect foresight of period $k$. If the cycle is W-stable, it is F-stable.

This result shows that given a mild assumption on the expectation function, namely that it detects not only period $k$ but also $2 k$, we should focus attention on those cycles of period $k$ that are asymptotically stable in the b.p.f. dynamics (1.4). A natural question then arises. Are all F-stable cycles of period $k$ also stable in the actual dynamics (1.3) ?

The answer is no, in general. Indeed, consider the simple example where $X$ is the real line, $T=3$ and

$$
\psi\left(x_{1}, x_{2}, x_{3}\right)=c x_{1}+(1-c) x_{3}
$$

Then $\psi$ detects period 2 . Consider now a stationary state, i.e. a fixed point $x$ of the map $F$, that is $F$-stable, 1.e. $a=F^{\prime}(x)$ has a modulus less than 1 . Linearizing $(1.3)$ at the stationary state, we get the characteristic equation

$$
\lambda^{3}-a\left(c \lambda^{2}+1-c\right)=0
$$

W-stability means that all roots of this equation lie within the unit circle. Note that for $c=-3$ and $a=1, \lambda=-2$ is a solution. Thus, when $c=-3$ and $a$ is less but close to 1 , the stationary state is F-stable but W-unstable.

We exhibit below (Theorem 5.1), in a slight generalization of Grandmont [8, Proposition 3.2], a restricted class of expectations functions for which all cycles of a given period $k$ that are $F$-stable are also $W$-stable. To predict $x_{t+1}$, these expectations functions form essentially locally an average of all past $k$-th observations, 1.e. of $x_{t+1-k}, x_{t+1-2 k}, \ldots$, and thus neglect intermediate data. Moreover, the weights have to verify a number of restrictions - which will be in particular satisfied if all weights are nonnegative. 
The paper is organized as follows. Section 2 defines precisely the notion of stability of a cycle. Section 3 describes the properties of the expectations functions that preserve cycles of a given period. The main Theorem is proved in Section 4. Finally, Section 5 describes a class of expectations functions ensuring that all F-stable cycles of a given period are also W-stable.

\section{STABILITY OF A CYCLE}

Consider a cycle with perfect foresight $\left(\bar{x}_{t}\right)$ of a period $k$ that is detected by $\psi$. Let $x_{1}^{*}, \ldots, x_{k}^{*}$ be the $k$ consecutive values taken by the state variable along the cycle (1.e. its periodic orbit), with $x_{s}^{*}=F\left(x_{s+1}^{*}\right)$ for $s=1, \ldots, k-1$ and $x_{1}^{*}=F\left(x_{k}^{*}\right)$. We wish to define the stability of this cycle first in the b.p.f. dynamics $(1.4)$ and then in the actual dynamics $(1.3)$.

Each $x_{s}^{*}$ is a fixed point of the $k$-th iterate of $F, x_{s}^{*}=F^{k}\left(x_{s}^{*}\right)$ and $x_{s}^{*} \neq F^{j}\left(x_{s}^{*}\right)$ for $j=1, \ldots, k-1^{(3)}$. We say that the cycle is $\underline{F-s t a b l e ~ i f ~ t h e ~}$ absolute value of the derivative of $F^{k}$, evaluated at $x_{s}^{*}$, is less than unity, i.e. $\left|\operatorname{DF}^{k}\left(x_{s}^{*}\right)\right|<1$. If we set for each $i=1, \ldots, k$

$$
a_{s}=F^{\prime}\left(x_{s+1}^{*}\right)
$$

we obtain from the chain rule of differentiation

$$
D F^{k}\left(x_{s}^{*}\right)=a_{1} \ldots a_{k}
$$

and we see that the stability condition $\left|a_{1} \ldots a_{k}\right|<1$ is independent of the 
particular point $x_{s}^{*}$ chosen on the periodic orbit.

We turn now to the definition of W-stability. First note that (1.3) determines a difference equation from $X^{\top}$ into itse if through the map $\tilde{W}^{z}$ that associates to any member $a_{t}=\left(x_{t-1}, \ldots, x_{t-T}\right)$ of $x^{\top}$ the element

$$
a_{t+1}=\left(W\left(x_{t-1}, \ldots, x_{t-T}\right), x_{t-1}, \ldots, x_{t-T+1}\right) \text {. }
$$

Clearly, the cycle is a periodic solution of (1.3) if and only if, for all $s=1, \ldots, k, a_{s}^{*}=\left(x_{s-1}^{*}, \ldots, x_{1}^{*}, x_{k}^{*}, \ldots\right)$ is a fixed point of the $k$-th iterate of $\tilde{W}^{z}$, i.e. $q_{s}^{*}=\tilde{W}^{z k}\left(q_{s}^{*}\right)$ and $q_{s}^{*} \neq \tilde{W}^{z j}\left(q_{s}^{*}\right)$ for $j=1, \ldots, k-1$. By definition, the cycle $\left(\bar{x}_{t}\right)$ is $\underline{W-s t a b l e}$ if all eigenvalues of the Jacobian matrix of $\tilde{W}^{k}$ evaluated at $q_{s}^{*}$, i.e. $D W^{* k}\left(q_{s}^{*}\right)$, have a modulus less than 1 . Here again, the definition does not depend upon the particular point chosen on the periodic orbit, for the Jacobian matrices $D W^{* k}\left(q_{s}^{*}\right)$ and, say, $D W^{k}\left(q_{1}^{*}\right)$, have the same eigenvalues ${ }^{(4)}$

The remainder of this section looks for a convenient way to find the eigenvales of $D W^{* k}\left(q_{1}^{*}\right)$, in terms of the function' $F$ and the expectations function $\psi$.

From the chain rule of differentiation applied to $\tilde{W}^{k}\left(q_{1}^{*}\right)=\tilde{W}\left(w^{k-1}\left(q_{1}^{*}\right)\right)$, one gets that $D W^{* k}\left(q_{1}\right)$ is the product of the Jacobian matrix of $\tilde{W}$ evaluated at the different $q_{s}^{*}=s=1, \ldots, k$

$$
D \tilde{W}^{k}\left(q_{1}^{*}\right)=D \tilde{W}\left(q_{k}^{*}\right) \ldots D \tilde{W}\left(q_{1}^{*}\right)
$$


The eigenvalues $\lambda$ of $D \tilde{W}^{k}\left(q_{1}^{*}\right)$ are therefore the solutions of the equation, in matrix notation

$$
\left[D \tilde{W}\left(q_{k}^{*}\right) \ldots D \tilde{W}\left(q_{1}^{*}\right)\right] v=\lambda v
$$

in which $v \neq 0$ is an eigenvector.

$$
\text { For every } \begin{aligned}
s & =1, \ldots, k, \text { and } h=1, \ldots, T \text {, let } \\
c_{h}^{s} & =\psi_{h}^{\prime}\left(x_{s-1}^{\star}, \ldots, x_{1}^{*}, x_{k}^{\star}, \ldots\right)
\end{aligned}
$$

be the partial derivative of $\psi$ with respect to $x_{t-h}$, evaluated at the point $q_{s}^{*}$ (if $k=1$, we drop the superscript and write simply $c_{h}$ ). In view of the fact that $W=F \circ \Psi$, for every $T$-dimensional vector $y$, the vector $u=D W\left(q_{s}^{*}\right) y$ is, with this notation, given by

$$
\begin{aligned}
& u_{1}=a_{s h=1} \sum_{h}^{T} c_{h}^{s} y_{h} \\
& u_{h}=y_{h-1} \quad \text { for } h=2, \ldots, T .
\end{aligned}
$$

For every $h \geq 2$, the component $u_{h}$ is equal to the lagged component $y_{h-1}$. When applied successively $k$ times, this fact yields that the equation corresponding to the $h$-th row of $(2.4)$ is for $h=k+1, \ldots, T$

$$
v_{h-k}=\lambda v_{h}
$$

On the other hand, it is easily seen from (2.6) that the k-th row of (2.4) reads

$$
a_{1} \sum_{h=1}^{T} c_{h}^{1} v_{h}=\lambda v_{k}
$$


As for the $(k-1)-$ th row, it yields

$$
a_{2} c_{1}^{2}\left(a_{1} \Sigma_{1}^{\top} c_{h}^{1} v_{h}\right)+a_{2} \Sigma_{2}^{\top} c_{h}^{2} v_{h-1}=\lambda v_{k-1}
$$

which can be rewritten by using (2.8)

$$
a_{2} \sum_{1}^{T} c_{h}^{2} v_{h-1}=\lambda v_{k-1}
$$

provided that $v_{0}$ is generated by using (2.7) for $h=k$, i.e. $v_{0}=\lambda v_{k}$. Pursuing recursively along this line, one finds finally that the equation corresponding to the row $(k+1-s)$ in $(2.4)$, for $s=1, \ldots, k$, is given by

$$
\text { a } \varepsilon_{1}^{T} c_{h}^{s} v_{h+1-s}=v_{1-s}
$$

in which $v_{0}, \ldots, v_{1-k}$ are obtained from the components of the efgenvector $v$ through (2.7), for $h=1, \ldots, k$.

To sum up, solving (2.4) for $\lambda$ and $v$ is equivalent to find $\lambda$ and $v_{1-k}, \ldots, v_{0}, v_{1}, \ldots, v_{T}$ that satisfy $(2.7)$ for $h=1, \ldots, T$ and $(2.10)$ for $s=1, \ldots, k$. This formulation makes clear what is actually done when solving (2.4) for $\lambda$ and $v$. One "linearizes" first the dynamics (1.3) around each point $q_{s}^{*}$ : this is represented by the Jacobian matrix $\underset{D W^{*}}{\left(q_{s}^{*}\right)}$ and by equation $(2.10)$. A complete linearized dynamics around the cycle is obtained by assuming that each linearized dynamics $(2.10)$ is "visited" consecutively from $s=1$ to $s=k$, and periodically, with period $k$. Then one looks for solutions of this complete linearized dynamical system that grow at the (possibly complex) rate $\lambda-1$ every $k$ periods (this is equation $(2.7)$ ), the efgenvector $v$ being interpreted as the initial position of the solution. 
If we are looking for solutions of (2.7), (2.10) such that $\lambda \neq 0$, we may without loss of generality choose to work with the unknowns $\lambda$ and $v_{1-k}, \ldots, v_{0}$ alone, since the other values $v_{1}$ through $v_{T}$ can then be obtained by using (2.7). It is now not difficult to verify that with these unknowns, the system (2.10) becomes

$$
\begin{aligned}
& {\left[1-a_{1} \gamma_{k}^{1}(\lambda)\right] v_{0}-a_{1} \gamma_{k-1}^{1}(\lambda) \quad v_{-1} \ldots \ldots-a_{1} \gamma_{1}^{1}(\lambda) \quad v_{1-k}=0} \\
& -a_{2} r_{1}^{2}(\lambda) \quad v_{0}+\left[1-a_{2} r_{k}^{2}(\lambda)\right] \quad v_{-1} \cdots \cdots-a_{2} r_{2}^{2}(\lambda) \quad v_{1-k}=0 \\
& -a \lambda \gamma_{k-1}^{k}(\lambda) v_{0}-a_{k} \lambda \gamma_{k-2}^{k}(\lambda) \quad v_{-1} \cdots+\left[1-a_{k} \gamma_{k}^{k}(\lambda)\right] v_{1-k}=0
\end{aligned}
$$

in which for every $r, s=1, \ldots, k$.

$$
\gamma_{r}^{s}(\lambda)=\sum_{h \geqslant 1}^{[} c_{(h-1) k+r}^{s} \lambda^{-h}
$$

(if $k=1$, we write simply $r(\lambda)$ ).

This system has a solution if and only if the determinant of the matrix composed of its coefficients, say $Q(\lambda)$, is zero. Thus the eigenvalues $\lambda$ of the Jacobian matrix $D W^{* k}\left(a^{*}\right)$ such that $\lambda \neq 0$ are the solutions of the equation $O(\lambda)=0$. It follows that the cycle $\left(\bar{x}_{t}\right)$ is $W$-stable if and only if these solutions verify $|\lambda|<1$. We have therefore proved:

PROPOSITION 2.1. Consider a cycle $\left(\bar{x}_{t}\right)$ with perfect foresight, with a perfod $k$ that is detected by the expectation function. Let

$\left\langle x_{1}^{*}, \ldots, x_{k}^{*}\right\rangle$ be the oeriodic orbit, with $x_{s}^{*}=F\left(x_{s+1}^{*}\right)$. 
The cycle is $W$-stable if and only if the solutions of

$$
Q(\lambda) \equiv \operatorname{det}[I-A \Gamma(\lambda)]=0
$$

\section{all lie inside the unit circle of the complex plane, where}

1) $A$ is the diagonal matrix

$$
\left[\begin{array}{lll}
{ }^{1} & & \\
& & \\
& \cdot & \\
& & a_{k}
\end{array}\right] \text { with } \quad a_{s}=F^{\prime}\left(x_{s+1}^{*}\right)
$$

2) $\Gamma(\lambda)$ is the matrix

$$
\left[\begin{array}{ccc}
\gamma_{k}^{1}(\lambda) & \gamma_{k-1}^{1}(\lambda) & r_{1}^{1}(\lambda) \\
\lambda r_{1}^{2}(\lambda) & \gamma_{k}^{2}(\lambda) & r_{2}^{2}(\lambda) \\
\vdots & \vdots & \vdots \\
\lambda r_{k-1}^{k}(\lambda & \lambda r_{k-2}(\lambda) & r_{k}(\lambda)
\end{array}\right]
$$

with, for $s=1, \ldots, k$ and $h=1, \ldots, T$

$$
c_{h}^{s}=\psi_{h}^{\prime}\left(x_{s-1}^{*}, \ldots, x_{1}^{*}, x_{k}^{*}, \ldots\right)
$$

and for $r, s=1, \ldots, k$

$$
r_{r}^{s}(\lambda)={ }_{h \geqslant 1}^{\sum} c_{(h-1) k+r}^{s} \lambda^{-h}
$$

\section{DETECTING CYCLES}

The foregoing proposition shows how the local properties of the b.p.f. dynamics $F$ and of the expectation function $\psi$ interact in a neighborhood of a 
cycle - through the matrices $A$ and $\Gamma(\lambda)$, respectively - as far as $W$ stability is concerned. Up to now, we have not fully exploited the fact that the expectations functions detects perfod $k$. It turns out that this property imposes strong restrictions on the behaviour of $\Gamma(\lambda)$ on the unit circle, which will be crucial for our main result.

LEMMA 3.1. Let $x=\left(x_{1}, \ldots, x_{T}\right)$ be a sequence in $x^{\top}$ that has period $k$. Let $c_{h}=\psi_{h}^{\prime}\left(x_{1}, \ldots, x_{T}\right)$ for $h=1, \ldots, T$ and define for every complex number $\lambda \neq 0$

$$
r(\lambda)=\varepsilon_{h} c_{h} \lambda^{-h}
$$

Then if $\psi$ detects period $k$

$$
\gamma(\lambda)=\lambda \text { when } \lambda=e^{2 \pi i d / k} \text {, all } d=1, \ldots, k
$$

Proof : Let $z=\left(z_{1}, \ldots, z_{T}\right)$ be a real vector with period $k$. If the real $\mu$ is small enough, $x+\mu z$ belongs to $x^{\top}$. If $\psi$ detects period $k$

$$
\psi(x+\mu z)=\psi(x)+\mu f(z)
$$

in which $f(z)=z_{k-1}$ if $k \geqslant 2$ and $z$, otherwise. Next, there exists $\bar{\mu}$ in $[0, \mu]$ such that

$$
\psi(x+\mu z)-\psi(x)=\mu \sum_{h} \Psi_{h}^{\prime}(x+\bar{\mu} z) z_{h}
$$

in which case

$$
\varepsilon_{h} \psi_{h}^{\prime}(x+\overline{\mu z}) z_{h}=f(z)
$$

Letting $\mu$ go to 0 yields

$$
r_{h} c_{h} z_{h}=f(z)
$$


The result follows by applying this relation to $z=(\cos 2 \pi \mathrm{dh} / \mathrm{k})$ and to $z=(\sin 2 \pi d h / k)$, for $d=1, \ldots, k$.

The next fact states the implications of the previous lemma for the expressions $\gamma_{r}^{s}(\lambda)$ appearing in Proposition 2.1, in the relevant case $k \geqslant 2$.

LEMMA 3.2. Let $x$ and $\gamma(\lambda)$ be as in Lemma 3.1 with $k>2$. Define for $r=1, \ldots, k$, and every complex number $\lambda \neq 0$

$$
\gamma_{r}(\lambda)=\sum_{h \geqslant 1} c_{(h-1) k+r} \lambda^{-h}
$$

Then if $\Psi$ detects period $n k, n \geqslant 1$

$$
\begin{aligned}
& \gamma_{r}(\lambda)=0 \text { for } r \neq k-1 \text { and } \gamma_{k-1}(\lambda)=1, \\
& \text { when } \lambda=e^{2 \pi / d / n}, \text { all } d=1, \ldots, n .
\end{aligned}
$$

Proof : The first step of the proof is to remark that

$$
k \lambda^{k-r} \gamma_{r}\left(\lambda^{k}\right)=\sum_{u=1}^{k} w_{u}^{r} r\left(\lambda w_{u}\right)
$$

in which $w_{u}=e^{2 \pi i u / k}$. Indeed, the right hand of $(3.3)$ is equal to

$$
\sum_{u=1}^{k} \sum_{h} c_{h} \lambda^{-h} w_{u}^{r-h}
$$

But $\sum_{u=1}^{k} w_{u}^{r-h}$ is equal to $k$ if $r=k$ (modulo $k$ ) and to 0 otherwise. Thus

$$
\begin{aligned}
\sum_{u=1}^{k} \omega_{u}^{r} r(\lambda \omega) & =k \sum_{h \geqslant 1}^{[} c_{(h-1) k+r} \lambda^{-(h-1) k-r} \\
& =k \lambda^{k-r} \gamma_{r}\left(\lambda^{k}\right)
\end{aligned}
$$

Rewrite (3.3) as 


$$
k \lambda^{(k-r) / k} \gamma_{r}(\lambda)=\sum_{u=1}^{k} w_{u}^{r} \gamma\left(\lambda^{1 / k} \omega_{u}\right)
$$

and assume that $\psi$ detects period $n k$. Let $\lambda=e^{2 \pi i d / n}$ for some $d=1, \ldots, n$. Then $\left(\lambda{ }_{u}^{1 / k} \omega_{u}\right)^{n k}=1$ and thus from Lemma 3.1 (applied to period $n k$ )

$$
r\left(\lambda^{1 / k} \omega_{u}\right)=\lambda^{1 / k} \omega_{u}
$$

One gets therefore for such a $\lambda$

$$
k \lambda^{(k-1-r) / k} \gamma_{r}(\lambda)=\sum_{u=1}^{k} w_{u}^{r+1}
$$

The result follows from the fact that the right hand of the last equality is equal to $k$ when $r=k-1$, and to 0 otherwise.

Remark 3.3. It can be shown that if $\psi$ is linear, then (3.1) (resp. (3.2)) is a necessary and sufficient condition for $\psi$ to detect period $k$ (resp. nk).

\section{PROOF OF THEOREM 1.1}

We are now in a position to prove our main result. Lemmas 3.1 and 3.2 yield the following important information concerning the value of $Q(\lambda)$ in Proposition 2.1 when the expectation function detects period $n k, n \geqslant 1$, and for $\lambda$ equal to a complex $n$-th root of unity.

LEMMA 4.1. Assume in Proposition 2.1 that $\psi$ detects oeriod $n k, n \geqslant 1$. Then

$$
Q(\lambda)=1-\left(a_{1} \ldots a_{k}\right) \lambda
$$

for $\lambda=e^{2 \pi i d / n}, d=1, \ldots, n$. 
Proof : If $k=1$, the cycle is actually a fixed point $x^{*}$ of $F$. The matrix A reduces to the single number $a=F^{\prime}\left(x^{*}\right)$, while $\Gamma(\lambda)$ reduces to its top left e lement $\gamma(\lambda)$. Thus

$$
Q(\lambda)=1-a r(\lambda)
$$

The result follows in that case by applying Lemma 3.1 to the period $n$.

If $k>2$, the result is a direct consequence of Lemma 3.2. It suffices indeed to remark that when $\lambda=e^{2 \pi i d / n}$, the matrix I - Ar $(\lambda)$ becomes

$$
\left[\begin{array}{ccccc}
1 & -a_{1} & 0 & \cdots & 0 \\
0 & 1 & -a_{2} & \cdots & 0 \\
\ldots \ldots \ldots & \ldots & \ldots & \ldots & \\
0 & 0 & 0 & \ldots & a_{k-1} \\
-\lambda a_{k} & 0 & 0 & \cdots & 1
\end{array}\right]
$$

Q.E.D.

Theorem 1.1 , which asserts that W-stability implies F-stability of a cycle with perfect foresight of period $k$ whenever the expectation function detects period $2 k$, becomes now a simple consequence of Proposition 2.1 and of the previous Lemma. Indeed,

PROPOSITION 4.2. Under the assumptions of Proposition 2.1, let $a=a_{1} \ldots a_{k}$. Then

1) If $a=1$, then $Q(1)=0$. If $a>1$, then there exists a real number $\lambda>1$ such that $Q(\lambda)=0$.

2) Assume that $\Psi$ detects $2 k$. Then $Q(-1)=0$ when $a=-1$. If $a<-1$, then there exists a real number $\lambda<-1$ such 
that $Q(\lambda)=0$.

Proof : First remark that, from Lemma 4.1, $Q(1)=1-a$. Thus $Q(1)=0$ if $a=1$. Now if $a>1$, then $Q(1)<0$. But if $\lambda$ is rea $1, Q(\lambda)$ is real and tends to 1 when $\lambda$ diverges to $+\infty$ (all elements of $\Gamma(\lambda)$ tend to 0$)$. Thus if a $>1$, there exists by continuity a real number $\lambda>1$ such that $Q(\lambda)=0$.

The argument is similar when $\psi$ detects period $2 k$. From Lemma 4.1 , we have then $Q(-1)=1+a$. Thus $Q(-1)=0$ when $a=-1$. If $a<-1$, one has $Q(-1)<0$, and by the same continuity argument as before, there is a real number $\lambda<-1$ such that $Q(\lambda)=0$.

Proposition 4.2 establishes indeed the validity of Theorem 1.1. If the expectation function detects period $2 k$, and if a cycle with perfect foresight of period $k$ is $W$-stable, then from Proposition 2.1, all solutions of $Q(\lambda)=0$ must lie inside the unit circle of the complex plane. Proposition 4.2 implies that one must have $\left|a_{1} \ldots a_{k}\right|<1$ in such a case. This is precisely the definition of F-stability.

\section{A RESTRICTED CLASS OF EXPECTATIONS FUNCTIONS}

Theorem 1.1 states that if $\psi$ detects period $2 k$, any cycle of period $k$ that is $W$-stable is $F$-stable. The example given in the introduction shows that the converse is not generally true. This indicates that one needs much more stringent conditions on the expectation function - specifically, on the behaviour of the matrix $r(\lambda)$ appearing in Proposition 2.1 on the unit circle to guarantee that F-stability leads to $W$-stability. A set of such conditions is provided by the following

THEOREM 5.1. Let $\left(\bar{x}_{t}\right)$ be a cycle satisfying the conditions of Proposition 2.1.

1) If $k=1$, let $\alpha \geqslant 1$ be the maximum of $|\gamma(\lambda)|$ on the 
unit circle. Then the stationary state is $W$-stable if $1-|a| \alpha>0$. In particular when $\alpha=1$, F-stability implies W-stability.

2) If $k \geqslant 2$, assume $\gamma_{r}^{s}(\lambda)=0$ for $r \neq k-1$ and $|\lambda|=1$, and let $\alpha_{s} \geqslant 1$ be the maximum of $\left|\gamma_{k-1}^{s}(\lambda)\right|$ on the unit circle, for $s=1, \ldots, k$. Then the cycle is W-stable if $1-\left|a_{1} \ldots a_{k}\right|\left(\alpha_{1} \ldots \alpha_{k}\right)>0$. In particular if $\alpha_{s}=1$ for all $s$, F-stability implies W-stability.

Proof : Consider first the case of a fixed point $x^{*}$ of $F$, with $a=F^{\prime}\left(x^{*}\right)$. Applying Proposition 2.1, we get the equation

$$
Q(\lambda)=(1-a \gamma(\lambda))=0
$$

Let $\alpha$ be the maximum of $|\gamma(\lambda)|$ for $|\lambda|=1$. One has $\alpha \geqslant 1$ since $\gamma(1)=1$ (Lemma 3.1). Remark next that $Q(\lambda)=1$ when $a=0$, and thus that all eigenvalues of $D \tilde{W}^{*}\left(q^{*}\right)$ are 0 . Note also that these eigenvalues vary continuously with the parameter a. Suppose now that 1) is false, i.e. that there is some a with $1-|a| \alpha>0$ such that the corresponding equation $Q(\lambda)=0$ has a root with $|\lambda| \geqslant 1$. In that case, there must exist $\bar{a}$ in $(0, a)$ such that the assoclated equation $Q(\lambda)=0$ has a root $\bar{\lambda}$ on the unit circle. One gets then

$$
|\gamma(\bar{\lambda})|=|/| \bar{a}|\geqslant| /|\mathbf{a}|>\alpha
$$

a contradiction. If $\alpha=1$, it is clear that F-stability $(|a|<1)$ implies W-stability.

The case $k \geqslant 2$ is dealt with by a similar argument. Under the assumption of 2), by a reasoning analogous to the proof of Lemma 4.1 , one gets 


$$
Q(\lambda)=1-\left(a_{1} \ldots a_{k}\right) \lambda \gamma_{k-1}^{1}(\lambda) \ldots \gamma_{k-1}^{k}(\lambda)
$$

The remainder of the proof is the same as in the case $k=1$, by a continuity argument on the vector $\left(a_{1}, \ldots, a_{k}\right)$.
Q.E.D.

It should be noted that when $k \geqslant 2$, the assumption $\gamma_{r}^{s}(\lambda)=0$ when $|\lambda|=1$ for $r \neq k-1$ means that all the corresponding coefficients $c^{s}(h-1) k+r, h \geqslant 1$ are equal to zero. This condition is thus very restrictive. It says that locally, the expectations function is an average of every $k$-th past observation. Theorem 5.1 above states in effect sufficient conditions of the weights ensuring that F-stability implies W-stability.

Remark 5.2. If all the coefficients of $\gamma(\lambda)$ when $k=1$, of $\gamma_{k-1}^{s}(\lambda)$ otherwise, have the same sign, they must be all nonnegative and sum to one since $r(1)=1$ or $\gamma_{k-1}^{s}(1)=1$ from Lemma 3.1 or 3.2 . One has clearly then $|\gamma(\lambda)|<1$ or $\left|\gamma_{k-1}^{s}(\lambda)\right|<1$ on the unit circle, and F-stability implies $W$-stability. The result had been proved in this particular case by a different technique in Grandmont [8, Proposition 3.2]. 


\section{FOOTNOTES}

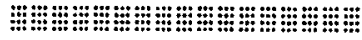

* CEPREMAP, 142, rue du Chevaleret, 75013 PARIS.

* INSEE, 18, Boulevard Ado iphe Pinard, 75675 PARIS CEDEX 14.

1. For details, see J. Geanakoplos and H. Polemarchak is [7], Grandmont [8], M. Woodford [9].

2. The restrictions to a fixed expectation function $\psi$ and a fixed (and finite) memory lag $T$ are there to enable us to deal with an autonomous (time independent), finite dimensional dynamical system. The assumption that $\psi$ does not depend on the current state $x_{t}$ is made for simplicity to avoid having to solve (1.1) (1.2) for $x_{t}$ in order to derive (1.3) below. The main results of the paper are preserved under a slight perturbation of the expectation function. They are thus still valid - provided that (1.1) (1.2) can be solved uniquely for $x_{t}$ - if $\psi$ does not depend "too much" of the current state variable $x_{t}$, a condition that is familiar in temporary equilibrium analysis.

3. The iterates of a function $f$ from a set into itself are defined recursively by $f^{\prime}=f, f^{j}=f \circ f^{j-1}$.

4. From the chain rule of differentiation applied to $W^{z k}\left(q_{f}^{*}\right)=W_{W}^{z}\left(W^{k-1}\left(q_{i}^{*}\right)\right)$, one gets that $D W^{k}\left(q_{i}^{*}\right)$ is the product of the Jacobian matrix of $\tilde{W}$ evaluated at each point $q_{1}^{*}, \ldots, q_{k}^{*}$,

$$
D W^{*}\left(q_{i}^{*}\right)=D \tilde{W}\left(q_{i-1}^{*}\right) \ldots D W^{\tilde{W}}\left(q_{i}^{*}\right) D W^{*}\left(q_{k}^{*}\right) \ldots D W^{*}\left(q_{i}^{*}\right)
$$

The statement follows then from the fact that for any two square matrices $A$ and $B$ with the same dimensions, the products $A B$ and $B A$ have the same eigenvalues. 
1. J. Benhabib and R. Day, Rational choice and erratic behaviour, Review of Economic Studies 48 (1981), 459-472.

2. J. Benhabib and R. Day, A characterization of erratic dynamics in the over lapping generations mode 1, Journal of Economics Dynamics and Control 4 (1982), 37-55.

3. P. Champsaur, On the stability of rational expectations equilibria, CORE DP 8324, Université Catholique de Louvain, Belgium.

4. G. Fuchs, Formation of expectations. A model in temporary general equilibrium theory, Journal of Mathematical Economics 4 (1977), 167-188.

5. G. Fuchs, Dynamics of expectations in temporary general equilibrium theory, Journal of Mathematical Economics 6 (1979), 229-252.

6. G. Fuchs, Are error learning behaviours stabilizing ?, Journal of Economic Theory 3 (1979), 300-317.

7. J. Geanakoplos and H. Polemarchakis, Walrasian indeterminacy and dynamic macroeconomic equilibrium : the case of certainty, mimeo, Yale University, 1983.

8. J.M. Grandmont, On endogenous competitive business cycles, Econometrica (1985), forthcoming.

9. M. Woodford, Indeterminacy of equilibrium in the over lapping generations model : a survey, mimeo, Columbia University, New York, 1984. 